\title{
The features of recovery muscle strength affected limb
}

\begin{abstract}
Conducted to test hypotheses about the role of somatic nociceptive afferentation in the recovery of muscle strength in the examined 80 patients children with closed fractures of the tibia in conditions of treatment according to Ilizarov, and the group of adult patients with osteoarthritis of the lower extremities (288 people). All subjects were examined using dynamometer to determine the maximum moments of force of the anterior and posterior groups of muscles of the thigh and Shin. It was found that the level of recovery of muscle contractility is higher in patients with children, forced to relieve pain syndrome to take painkillers for 1-2 days after injury, and the rate of bone regeneration-in taking pharmaceuticals for 3 days. The appearance of pain in the initial stages of osteoarthritis led to a compensatory increase in the contractility of the muscles of the affected limb in patients 20-35 years. The increase in the absolute strength of the muscles of the limb compared to the healthy stored at 2 stages of the disease.
\end{abstract}

Keywords: pain, bone fracture, osteoarthritis, muscle strength
Volume 6 Issue 2 - 2019

\section{SchurovVA}

The Federal State-Financed Institution, Russian Ilizarov Scientific Center for Restorative Traumatology and Orthopaedics, Russia

\begin{abstract}
Correspondence: Schurov VA, The Federal State-Financed Institution, Russian Ilizarov Scientific Center for Restorative Traumatology and Orthopaedics, Russia, Tel 73522454758, Emailshchurovland@mail.ru
\end{abstract}

Received: May 22, 2017| Published: April 05, 2019

\section{Introduction}

It is widely believed that any injury should lead to a violation of the functional abilities of the musculoskeletal system. At the same time, from ancient times known allopathic principle of treatment, which proclaimed Hippocrates, and says that the factors that can damage the body, and can contribute to its cure: contraria contraris curantur. This point of view is shared by some modern researchers. ${ }^{1}$ Of course, we are not talking about serious injuries and injuries of the musculoskeletal system.

At the time of injury, protective mechanisms - stress - limiting systems-come into effect. After that, there is the inclusion in the work of a larger number of reserve cells, which is accompanied by hypertrophy and hyperplasia of the relevant organs and tissues. ${ }^{2}$

It is known that under the influence of intensive training with weights, muscle hypertrophy develops. Along with the energy hypothesis of muscle hypertrophy due to hypoxia, the hypothesis of direct damage to muscle fibers is currently popular. ${ }^{3-5}$ The resulting damage is enhanced by the release of enzymes that enhance the manifestation of inflammatory reaction, swelling and pain. Reduction of the effect of increasing muscle strength as a result of adaptation to muscle activity and strengthening the effect after a break in the training process indicates the participation of the Central reflex link in the observed effect.

Osteoarthritis is a group of diseases of different etiology with similar biological, morphological and clinical manifestations and outcome, which are based on the defeat of all components of the joint. ${ }^{6}$ Osteoarthritis of the lower extremities is accompanied by a restriction of motor activity of patients, the appearance of pain at rest and with functional load, which inevitably should lead to a decrease in the contractility of muscles. ${ }^{7}$

There was a hypothesis that chronic somatic, including pain afferentation under certain conditions can also be an incentive for compensatory hypertrophy of the muscles of the limb.
The aim of the study is to test the hypothesis of the possibility of stimulating the impact of nociceptive afferentation on the subsequent development of muscle hypertrophy.

\section{Material and methods}

2 groups of patients were examined. The first group consisted of 80 children 3-17 years with closed fractures of the tibia in conditions of treatment according to Ilizarov. The duration of the pain syndrome was judged by the duration of application after injury of pain medications. The second group consisted of 288 patients aged 16 to 75 years with initial stages of knee joint damage. The intensity of pain syndrome was determined using a visual-analog scale.

The maximum moments of force of the anterior and posterior groups of thigh and Shin muscles were determined in all the subjects with the help of dynamometric stands ${ }^{8}$ developed by us.

Statistical processing of research results was carried out using the data analysis package "AtteStat". ${ }^{9}$ The normality of the distribution of empirical samples is confirmed by the modified Kolmogorov criterion. The tables show the average values of indicators and standard error. In the graphs of the error bar corresponds to the value of the standard deviation. In the analysis of the dynamics of changes in indicators, the methods of correlation and linear regression analysis were used. In order to take into account the effect of multiple comparisons, the analysis of variance using the Scheffe criterion for independent samples was applied. To assess the statistical significance of differences in the results in the case of analysis of two samples, student's t-test was used for independent samples.

\section{Results of the study and their discussion}

It was found that in patients of group 1, the duration of pain after a limb injury affects the duration of treatment. (Figure 1) The duration of the period of fixation of the bone was the least in shortterm pain syndrome and also for pain that needed to be stopped using pharmaceuticals during the first 4-5 days. The reduction of the terms 
of fusion with a relatively long pain syndrome may be a consequence of a more complete mobilization of protective and adaptive reserves of the body of children during this period.

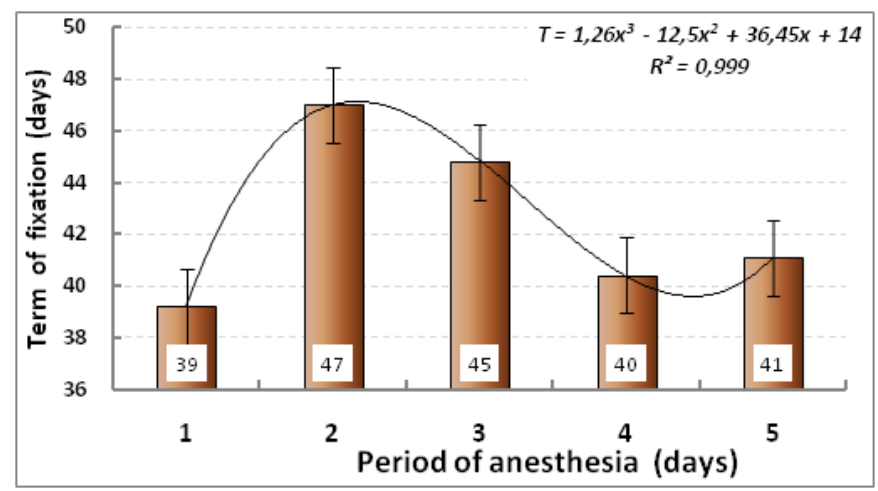

Figure I The relationship between the duration of pain medication and the duration of fixation of the limb in the llizarov apparatus.

Among the sick children, half were patients with a fracture of one tibia. The average duration of fixation at the fracture of 1 and 2 bones was 39-1.5 and 43-1.5 days, respectively. After the treatment of sick children, the contractility of the muscles of the damaged limb approached the level of the muscles of the intact limb. It turned out that the long-term pain syndrome had rather a negative impact on the level of recovery of muscle contractile properties. (Figure 2) The optimum duration of the pain syndrome to restore the contractility of the muscles was 1-2 days.

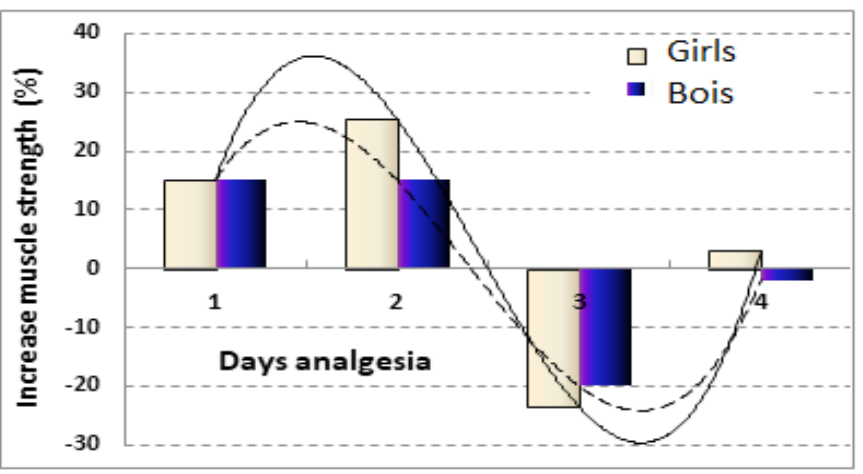

Figure $\mathbf{2}$ The ratio of muscle strength of the damaged and intact tibia in patients of different sexes in the long term after treatment for different duration of post-traumatic pain syndrome.

In patients of group 2 (with osteoarthritis), the ratio of the strength of the posterior group of muscles of the affected and intact tibia in 17 23 years was $88 \% \pm 4$, in $24-31$ years $111 \% \pm 5$ and in older age $90 \% \pm 3$. The increase in 24-31 was statistically significant ( $<<0.006$, Figure 3 ).

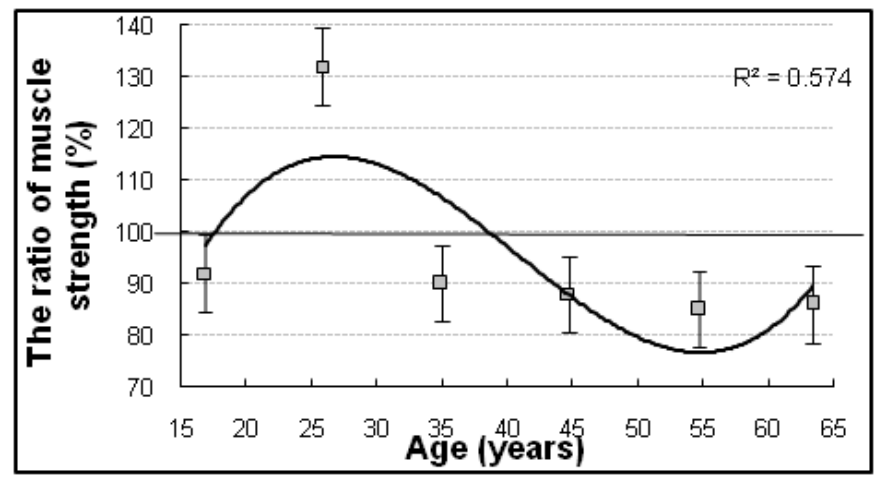

Figure 3 Age dynamics of the moment of force of the posterior group of the Shin muscles of the patient and intact limbs in patients with osteoarthritis.
As the age-related exhaustion of functional adaptation reserves after 31 years of compensatory increase in the strength of the muscles of the affected limb was not observed, moreover, the pathological process began to cover various joints of both limbs.

It should be noted that the name "intact limb" conditionally. The patient at the initial stages of the disease does not complain about the condition of this limb precisely because of the development of compensatory changes in it, in particular, an increase in the contractility of the muscles, most pronounced at the 2 nd stage of the disease. (Figure 4)

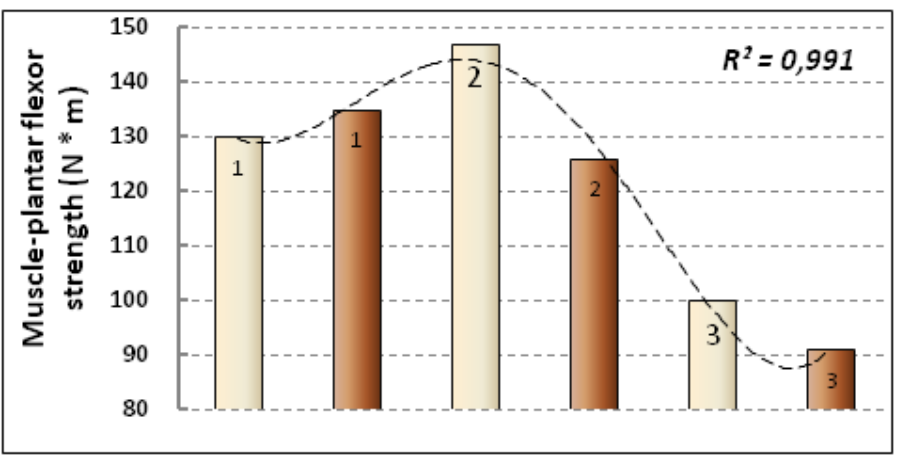

Figure 4 The moment of the force of the posterior muscle group of the tibia intact (less affected) and more affected limbs in patients with I, 2 and 3 stages of osteoarthrosis.

Thus, in the examination of patients with osteoarthritis, muscle atrophy and a decrease in the maximum strength are not so obvious and become noticeable, according to clinical studies, mainly in people over 35 years. It is assumed that there are a number of adaptivecompensatory mechanisms that allow patients to maintain locomotor activity and efficiency for a long time.

During phylogenesis mechanisms of damaged tissue restoration were developed, the rate of which depends on the strength and duration of the traumatic effect. ${ }^{10}$ Pain factor in bone injuries and limb joint disease is important as an indicator of the severity of the pathological process. However, when maintaining reserves of functional adaptation in young people with relatively small injuries or in the initial stages of the disease may indicate the possibility of maintaining and compensatory strengthening of the contractility of the muscles.

\section{Summary}

a. A quantitative study of the effect of gravity of the closed injuries of bones tibia in children in terms of treatment by Ilizarov allowed to reveal the stimulating effect of the arising of pain at the speed of the subsequent regenerative process of the bone and recovery of contractile capacity of muscle.

b. In the initial stages of the disease gonarthrosis in patients 2030 years possible compensatory increase in the contractility of the muscles of the affected limb. An increase in the absolute strength of the anterior group of thigh muscles of the affected limb is observed at the 2nd stage of gonarthrosis.

\section{Acknowledgments}

None.

\section{Conflicts of interest}

The authors declare there is no conflict of interest. 


\section{References}

1. Karlyshev VM. Wisdom of the disease. Bulletin of South Ural state University. 2001;3:24-25.

2. Masyutin VA, Vashetko RV, Shirokov DM. The possibility of assessing the functional reserves of the body in the early post-traumatic period. Traumatology Orthopedics of Russia. 1994;6:86-88.

3. Mak-Komac AJ. Skeletal muscles. Structure and functions. Kyiv: Olympic literature; 2001:407S.

4. Friden J, Lieder RL. Eccentric exercise-induced injuries to contractile and cytoskeletal muscle fibre components. Acta Physiol Scand. 2001;171(3):321-326.

5. Gibala MJ, Interisano AS, Tarnopolsky MA, et al. Myofibrillar disruption following acute concentric and eccentric resistance exercise in strengthtrained men. Can J Physiol Pharmacol. 2000;78(8):656-661.
6. Martynov AI. Internal diseases: textbook in 2t/ed. $3 \mathrm{rd}$ ed. ISPR, Moscow: GEOTAR-Media; 2005;2:814-822.

7. Forty NF, Yagur VE. Clinical study of joints in rheumatic diseases: hands for doctors. Minsk: Belarus; 2006:447.

8. Schurov VA. Dynamics of the biomechanical properties of the skin, artery walls and skeletal muscle increasing in the longitudinal size of the final. Russian Journal of Biomechanics. 2015;19(2):410-417.

9. Gaidyshev IP. Analysis and data processing. Special reference book. Sanct-Peterburg, Peter; 2001:752.

10. Schurov IV, Marfizin VP, Shved SI, et al. In the Phenomenon of anti entropic acceleration of recovery of functional and structural properties of tissues of injured tibia. Genius of Orthopedics. 2006:63-66. 